\title{
Don't be fazed by PHASER: Beginning exploration of a cyclical motivational system
}

\author{
JAMES T. TOWNSEND \\ Indiana University, Bloomington, Indiana
}

\begin{abstract}
An earlier article in this journal (Townsend, 1991) reviewed in general terms the PHASER software and manual. The present paper illustrates how PHASER may be used in investigation of a nonlinear dynamic psychological model and exhibits several of PHASER's facilities and attributes.
\end{abstract}

In preparing this paper, I could not resist the title, but in actuality, PHASER is not very intimidating. I might admit within confessional circumstances to being a computer cretin. I did my share of machine language programming in the late 1950 s and early 1960 s and went on to gain some minimal, if far from elegant, knowledge of the ALGOL and FORTRAN of the period. However, I have pretty much eschewed personal intimacy with computers ever since, not because I don't like them, but because I easily get addicted to them-like with detective stories, I never know how my program will turn out. My central and somewhat mystical belief is that computers should be our slaves and not our masters, and I easily backslide, given half a chance, to wallowing in computer licentiousness. It has only been in the last few years that I broke down and learned to use a word processor and e-mail and, of course, am now in danger of becoming a hopeless junkie once again.

The reason for this personal revelation is that I suspect that there are many out there like me, furtively masking their ignorance of the vast array of hardware and software arcana. Many investigators appreciate the incredible abilities of modern computers and all they can do for us but, somewhat defensively, hold the antiquated belief that experiments and theory per se are what should be driving our research, not the equipment itself. We are then brought to the topic of this paper, which specifically addresses a modest but useful software package and demonstrates the power that modern computer tools have to aid us in our modeling efforts. Here even a computer cretin like me can perform sleight of hand with an inexpensive program that would have been considered almost miraculous a couple of decades ago.

The present paper will not review the details of PHASER (Koçak, 1989), some of which were described in a previous article (Townsend, 1991) but will give an example of its use in constructing a dynamic model called MOT (Motivational Oscillatory Theory) and graphing its behavior. Some of the basic graphics tools will be illustrated in the context of that very simple model, but a few

Correspondence should be addressed to J. T. Townsend, Department of Psychology, Indiana University, Bloomington, IN 47405. of the more exotic instruments are best revealed with the aid of PHASER's canned routines.

First, a word about "dynamics." In the broad sense, any model or theory that describes behavior across time is dynamic. In that sense, a Turing machine is dynamic, a Markov chain is dynamic, and so on, and legitimately so. Most interesting dynamic theories posit a state space, that is, a set of states that can potentially be occupied by the system across time. The dynamic behavior is specified by a function or map from the present state, present input, and perhaps even output, to the next output and the next system state. The above types of systems conform to this model, albeit with probabilities attached in the case of Markov chains, as do finite and infinite state automata, probabilistic automata, and linear and nonlinear dynamics. It is interesting that many contemporary connectionist models are partly, but not totally, dynamic. For instance, many recent connectionist models give a dynamic prescription for learning across trials but lack a specification of within-trial, real-time dynamic information flow. This does not imply that such models are not highly useful, but only that a more complete description of their dynamic operation would be of considerable interest.

There is a more restricted definition of "dynamics" that is based on specification of the state transition function in terms of difference (discrete time) or differential (continuous time) equations. When this is done, one immediately makes contact with several hundred years of physics and the branches of differential equations that evolved to solve problems and derive laws of natural phenomena. The model investigated in this paper falls into this category, being defined by a small set of differential equations. Such systems may be deterministic or stochastic and involve partial derivatives (i.e., more than one independent variable, e.g., space as well as time) as well as ordinary derivatives and even mixtures of difference and differential and so-called integral equations. Furthermore, it is well to keep in mind that much can often be learned about very broad classes of systems even when they may not be specifiable in terms of such equations (but still the state transition function is present; see, e.g., Sibirsky, 1975). For more information on dynamic systems, the reader is referred to a multitude of books on differential 
equations and such modern "systems theory" works as Luenberger (1979), Beltrami (1987); Hirsch and Smale (1974); and Devaney (1986).

Let us begin by making clear the distinction between analytic or closed-form mathematical formulas, computer computations, numerical approximations, and computer simulations. These concepts are often confused in our literature. Analytic modeling implies carrying out derivations using explicit mathematical demonstration and ending in one or more explicit mathematical expressions. In many cases, the behavior of the model can be immediately evaluated from these expressions. However, in some instances, the expression may be so complex that computers are required to perform calculations using the expression in order to unveil the behavior-thus the second type of modeling. With nonlinear systems dynamics, the differential equations will be explicit and "analytic" but often cannot be explicitly solved. Because of this intractability, it is not even possible to employ the second type of modeling, that of perfect computation: numerical approximation is required. The routines in PHASER are of this ilk, and hence grave caution is demanded on the part of users. Finally, when the software mimics the model in real time, we say it is "simulating" the predicted behavior. A very common type of simulation is found with the usual Monte Carlo procedures. There are, of course, many situations where the dividing line between two or more of these strategies becomes thin to invisibility, but the distinctions are nonetheless important.

I do not consider our present model of oscillatory motivated behavior, MOT, to be final or complete. It was developed originally within the context of the dynamic decision field theory of Busemeyer and Townsend (1990, in press; Townsend, 1988; Townsend \& Busemeyer, 1989) to instantiate some of our ideas about motivated human decision making, in the hope of mimicking more or less natural cyclical milieus. It appears very simple indeed but is nevertheless nonlinear, and at this time, we do not have a complete handle on its behavior. We will use MOT here to illustrate nonlinear, but relatively simple, dynamic modeling using PHASER. The primary goal is to outline the way in which the scientist may begin to probe the behavior of a complex dynamic system. Along the way, limitations and advantages of the process will become clearer.

As noted in my review of PHASER, the latter is best employed to teach about nonlinear, and often exotically so, dynamics, using PHASER's canned routines. However, with care, PHASER can also be helpful in creating one's own initial dynamic models. The main limitation is that longer equations run off the screen and the programmer cannot see all of the typed-in symbols. Also, the editing is awkward in that if a mistake is made, the programmer must start the whole "sentence" over.

To begin investigation of cyclic behavior associated with such vacillating needs as eating on a simple system such as PHASER, I had to trim our theory down to those aspects most critical for the present circumstances: (1) the motivation state, motivation; (2) the consumption/satiation state, called consumption; (3) the preference state, preference; and (4) the action in real time and space, based on distance. The result is MOT, as presented in this paper (and in Townsend, 1988). Figure 1 shows MOT's architecture and flow.

In the traditional information-processing depictions, the flow is in discrete stages, or Donderian (after the 19th

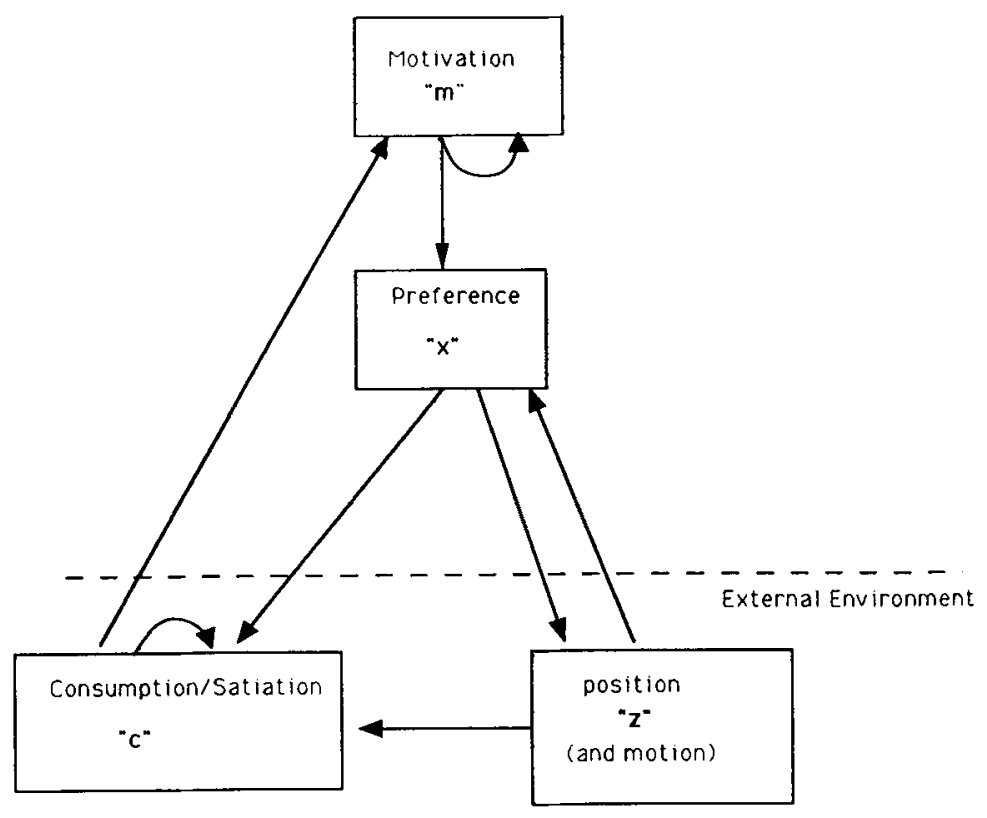

Figure 1. Signal-flow diagram for MOT, a continuous-flow, "motivational ascillatory theory." 
Century physiologist who pioneered in the use of reaction time to study mental processes), meaning that each stage completes processing before the next begins (e.g., see Townsend \& Ashby, 1983). In MOT, signals from one subsystem to the others flow continuously, with all the subsystems interacting at every instant.

In the more complete theory (e.g., Busemeyer \& Townsend, in press; Townsend \& Busemeyer, 1989), the motivational systems include approach and avoidance impulses, which are associated with various potential events. These systems are connected, via weights denoting importance, frequency, and attention, to valences identified with each possible choice alternative. The valences in turn drive the preference state through connection terms that represent the structure of the choice situation. Finally, the preference system provides the engine that actually moves, either at some point or continuously, to action. However, consumption and cyclicity were not previously visible in the published theory.

As in almost all useful dynamic models, the action in MOT takes place through explicit differential (or difference) equations. In the present scaled-down (but this time including consumption/satiation) theory, preference and valence are combined. Furthermore, here we consider only a single object of desire. Prospects for a richer environment will be considered later.

The primitive motivational rate of change is positive if the sum of current motivation, $m$, and state of satiation (associated with consumption), $c$, is less than some prescribed level of positive motivation, $M$. If that sum, $c+m$, is greater than $M$, then the rate of change of motivation is negative and motivation for the object decreases. The corresponding first order differential equation is then

$$
d m / d t=M-m-c \text {. }
$$

Note that this equation is linear in the state variables, $m$ and $c$.

Next, preference toward the object is governed by the level of motivation and the distance of the decision maker from the object. If motivation is positive, then the preferential state tends to increase in strength; the opposite is true if motivation is negative. And if the object is a long way from the decision maker, then the rate of change of preference is less than if it were close. The latter structure captures the approach-gradient-concept aspect of our theory, earlier advanced by Hull (1938), Lewin (1935), and Miller (1959). Letting $x=$ preference toward the goal, this leads to the expression

$$
d x / d t=[g(d)+1] \times m,
$$

where $g(d)$ is the preference gradient describing the way in which preference depends on the physical distance of the decision maker from the goal. In fact, for present purposes, we let $g(d)=1 /\left(z_{1}^{2}+z_{2}^{2}+a\right)$, a decreasing function of distance from the goal. Note that we could write $g(d)=1 /\left(d^{2}+a\right)$, where $d$ is in the Euclidean metric.

As for the consumption/satiation state, it is larger the stronger the preference variable and it decreases as the cumulated consumption increases. It also has a distance gradient attached to it and can be positive or negative.

A few words are appropriate here about the consumption/satiation state relative to the distance gradient. The distance gradient plays a different role here. In the real world, consumption often has an all-or-none threshold, starting only when the organism is in proximity to the goal object, for example, food or a sex goal. On the other hand, it is reasonable to model the other systems in a continuous mode. It can be awkward to work with hybrid, discrete-continuous systems through differencedifferential equations. Our approach here is to approximate such threshold shifts. For instance, one can approximate an absence of consummatory behavior except when the subject is close to the goal object, by the use of continuous functions that go to zero very rapidly as the distance from the object increases. This strategy is frequently employed in other areas of science and engineering. If $c=$ consumption/satiation variable, $C=$ pure consumption ceiling, and $h(d)=$ consumption distance function, then

$$
d c / d t=(x+C-c) \times h(d) .
$$

We set $h(d)=b /\left(z_{1}^{2}+z_{2}^{2}+r\right)$. This function is, of course, from the same family as $g(d)$ above, but now we can attempt to choose the parameters $b$ and $r$, so that $d c / d t$ is far from zero only when the decision maker is physically far from the goal, thus approximating the fact that consumption generally occurs for biological drives (as sometimes opposed to such derived motivations as the insidious, but well-documented, television drive).

The action in real-space coordinates, taken here to be two-dimensional, is described by a linear differential equation driving the decision toward or away from the goal according to the sign of the preference variable. Let $z=$ $\left(z_{1}, z_{2}\right)$, and without loss of generality, place the goal at $z=(0,0)$ so that $d z / d t=-x \times z$. This vector equation can be reexpressed in terms of the separate coordinates as $d z_{1} / d t=-x \times z_{1}$ and $d z_{2} / d t=-x \times z_{2}$.

The reader may observe that there are hardly any nonlinearities in MOT, the exceptions being the division of terms by the distance function (in consumption and preference) and the multiplication of the space coordinates, $z_{1}$ and $z_{2}$, by preference, $x$.

One of the annoying aspects of using numerical techniques to probe the behavior of dynamic systems is picking the proper initial conditions, that is, the starting points at $t=0$ for the dynamic variables of interest. In the present model, these variables are $c(t), x(t), z_{1}(t), z_{2}(t)$, and $m(t)$. Note that $d, h(d)$, and $g(d)$ can all be found from the other variables.

A closely allied difficulty-in fact, one that interacts with setting the initial conditions-is that of selecting appropriate parameter values. Unlike physics, where initial conditions and parameter values are usually prescribed by the situation, usually in psychology, the form of the functions is hypothesized in a "reasonable way." However, we often have little idea as to the "best" numbers 
to assign, especially for the parameters. Indeed, the basic system action, including asymptotic behavior, can be altered in nonlinear systems, simply by picking a different starting point or a slightly different set of parameters. Sometimes, theorems can be proved that confine the system to certain varieties of response for well-defined regions of initial conditions and parameter values; but this is not always possible or it may await intense expert mathematical effort.

We are still in the process of analyzing MOT as well as generalizing it, but a hint of the difficulties that can crop up can be seen in Figure 2, using the $X_{i}$ versus $t$ facility on PHASER. Here the initial conditions, pictorial window size and time frame allotted in PHASER, simply do not allow the system to reveal its long-term behavior. The upper graph shows a key variable, motivation evolving over time in a one-dimensional fashion. This type of plot represents the so-called "integral curve," that is, a graph of the solution of the differential equation. In this case, the solutions are approximated by PHASER. Note that time is explicit in this type of depiction, as opposed to "phase space" trajectories, in which time is implicit and invisible.

Motivation, consumption/satiation, and distance from the goal, $d$, are plotted in "phase space" in the bottom half of Figure 2, the latter being the state space of the system with time implicit. This view is called "PhasePort" in PHASER's menu. Curves in phase space (in two dimensions, often called the "phase plane") are referred to as "trajectories" or "orbits." Trajectories reveal how two or more of the state variables (e.g., motivation, preference, etc.) vary with one another over time, but time itself is not represented explicitly. Of course, we are restricted to two- or three-dimensional plots, and hence we are limited to the number of state variables we can simul- taneously depict. When the dynamic system requires more than four dimensions, as in the present model, the investigator uses PHASER to project the multidimensional trajectory down to a three-dimensional subspace.

The bottom plot is actually in three-dimensional perspective, although in the present case, one of the axes is invisible because it is coming directly at the viewer. In the plots against time, $t, m$ runs off the screen so we cannot see it throughout the plot. Below, we cannot tell how long it took for the trajectory to leave (partly because we are unable to effectively link up the top graph with the bottom). Our time range is too short and, apparently, the spatial window too narrow to obtain a realistic view of long-term behavior.

Figure 3 shows an improved view of behavior in this system. The four major aspects of the system state, including motivation $(m)$, distance from the goal $(d)$, state of preference $(x)$, and consumption/satiation are exhibited as a function in the top of the figure. Actually, in this and following figures, we simply plot one of the spatial coordinates $z$ instead of $d$. Since our metric, $d$, is symmetric in the coordinates, this does no harm. It is a general ability of PHASER to allow successive plotting of different functions on the same graph for the sake of comparison. This time we have selected space and window sizes that are more appropriate within the context of the current parameters and initial conditions. The labels of these variables are hand drawn, and in everyday usage the curves might be limned in color to aid visual analysis. Note the cyclicity that appears to be developing and the fact that the periodic functions do not appear to be sinusoids.

Observe that the distance is fluctuating between zero and a certain distance away from the goal and that in the present context, preference is oscillating but always

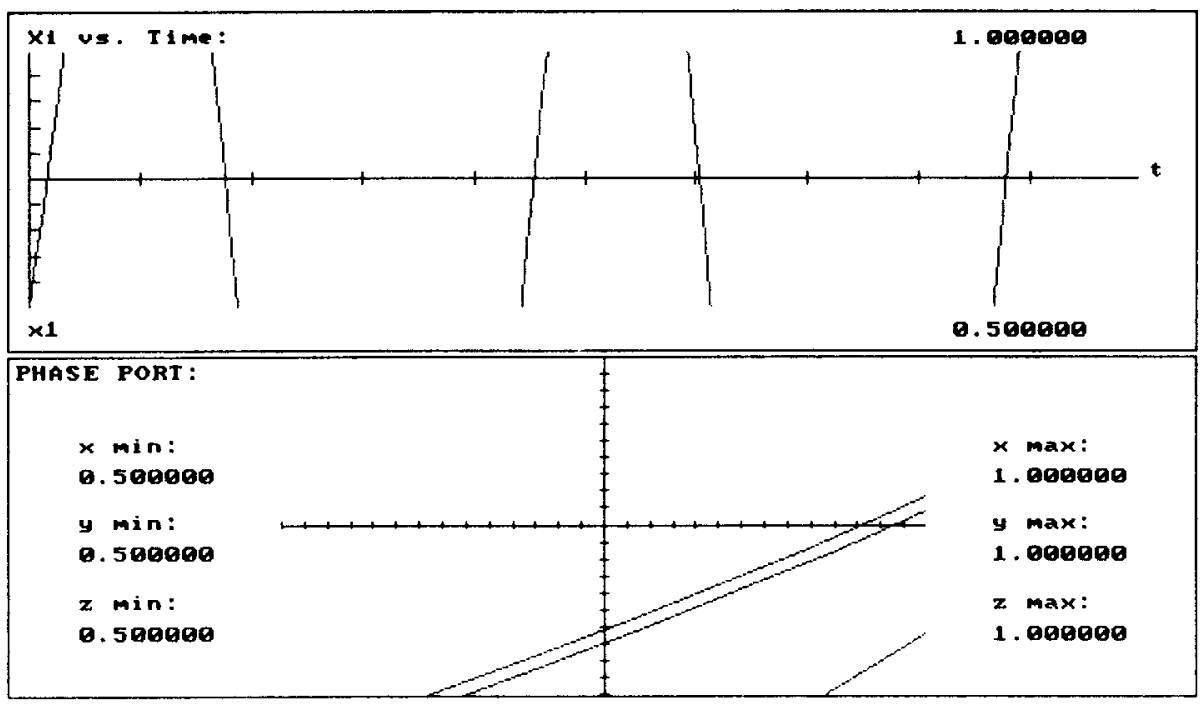

Figure 2. Illustration of the trouble one can get into when inappropriate space and time windows are chosen for one's graphics (see text for further discussion). 


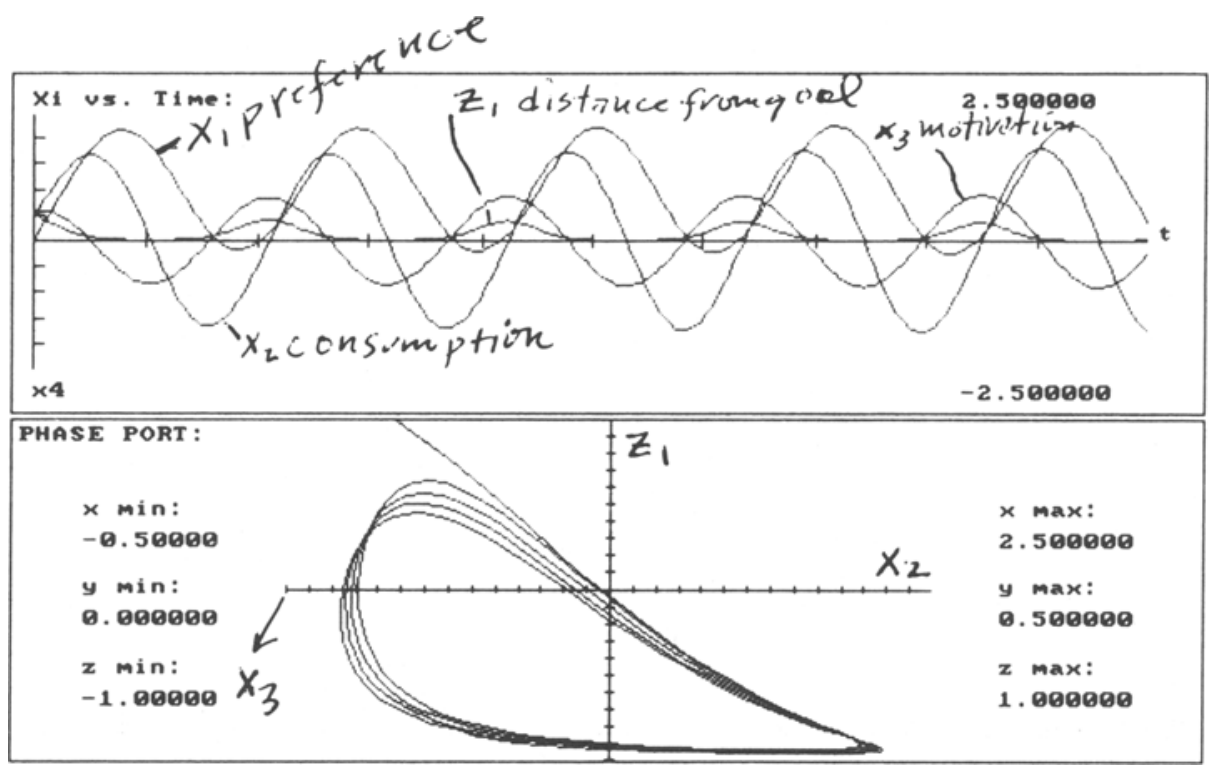

Figure 3. The top graph shows integral curves (numerical solution of the differential equations) for the four major state variables: motivation, preference, consumption/satiation, and distance from the goal. The bottom figure illustrates the same information, excluding preference, plotted in a threedimensional phase portrait trajectory.

greater than zero. Recall that preference provides the engine that moves the decision maker and is itself a function of motivation and the goal gradient (i.e., distance from the goal, increasing the closer one is to the goal). These aspects show up in Figure 3. However, although these four state variables are oscillating in a regular and generally reasonable way with one another, there are some facets that suggest further refinement should be carried out. Consumption is too slow a function of distance from the goal and hits its peak before preference reaches a maximum. Also, consumption does not reach its maximum at exactly the minimum distance from the goal, although this is a minor consideration if the overall behavior is appropriate.

The bottom of Figure 3 shows a phase portrait of a trajectory in three-dimensional space picturing consumption on the $x$-axis, distance from goal on the $y$-axis, and motivation on the $z$-axis, coming out toward the reader. Preference is missing in this graph. This figure reinforces the general conclusions from the time plot for the depicted variables: consumption reaches its peak at a small distance from the goal (perhaps not the very smallest), and motivation has declined as consumption has increased. Interestingly, although cyclical, the behavior for these three variables is nowhere near a limit cycle (see below). The motivational part of the trajectory seems to be in high flux from cycle to cycle, although the qualitative form of the cycle stays the same.

It now behooves us to take a detour to consider one of PHASER's "in-house" routines. Figure 4 includes the facility known as a "direction field," for PHASER's Hopf bifurcation (see Hopf in PHASER). Basically, this is just a plot of the vectors, which represent two of the deriva- tives (i.e., two of the differential equations). Hence, each vector denotes the tendency of motion (strictly speaking, the tangent to the surface of motion) for those two variables. The origin of each vector is, of course, the current state given by the current value of the two variables. PHASER does not draw in the "arrowheads" of the vectors to indicate actual direction; that must be done by the operator, as we did in Figure 4 for a few of them. Another limitation of PHASER is that it cannot plot direction fields for systems with more than two dimensions, such as our motivational model here. In principle, one could project down from many to two dimensions and then depict the direction field for those two dimensions. Perhaps this facility will be added at some point.

Within the direction field of Figure 4, we see four trajectories plotted by PHASER, each for a different set of initial conditions. Observe that two start outside the elliptical figure and two start inside. This suggests the appearance of a "limit cycle," wherein all trajectories in the region approach a periodic function. If the initial conditions corresponded to a point on the cycle itself, the trajectory would never leave that closed curve. Basically, the limit cycle acts like a fixed, asymptotically stable fixed point, that is, a "point attractor," which is approached ever more closely by nearby trajectories. But, in this case, the attractor is a set of points representing a periodic trajectory. A limit cycle cannot be produced by a linear system, but unlike some of the more exotic aspects of nonlinear dynamics, has long been known and used in physics and engineering.

The origin in Figure 4 is itself a fixed point, also called an equilibrium point, as noted above. If such a point were chosen as the initial condition, then there would be no 


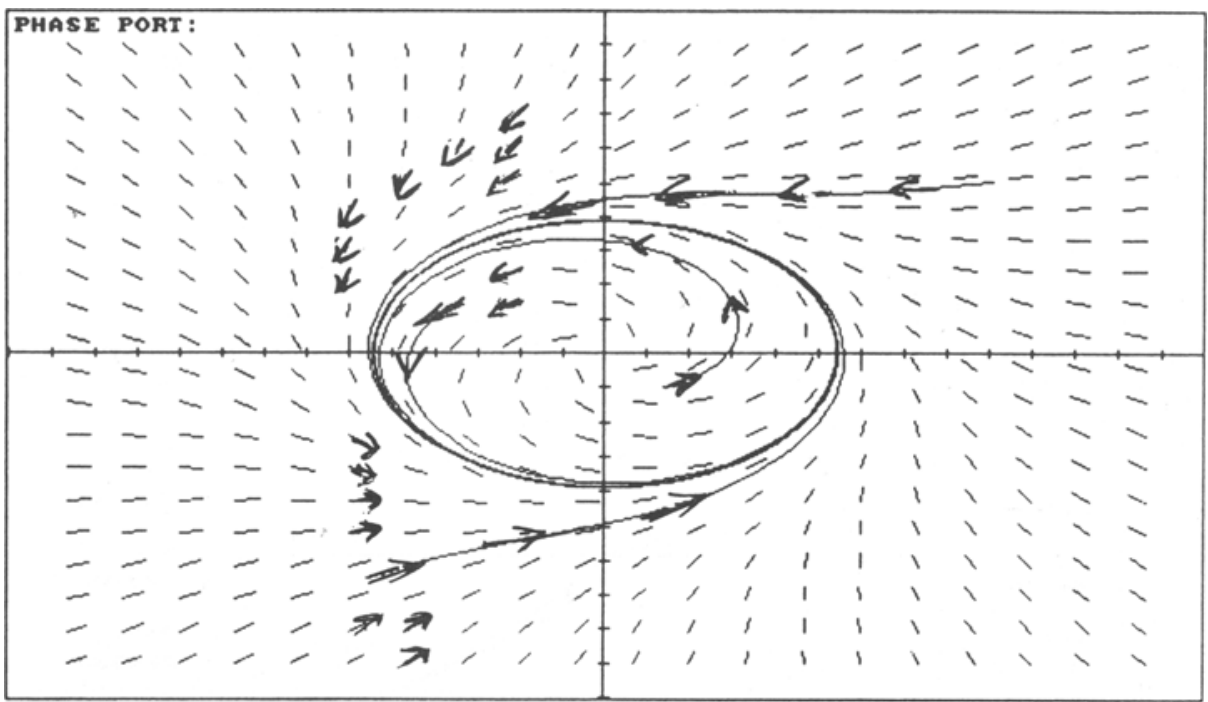

Figure 4. Limit-cycle behavior with four initial conditions in a two-dimensional phase portrait, along with the generating vector direction field. Plotted from Hopf in PHASER.

motion whatsoever for the depicted variables. In typical cases that are similar to Figure 4, such an equilibrium point inside a limit cycle is "unstable" in that if the initial starting point is even a tiny bit different from it, the trajectory will lead away from the equilibrium and toward the limit-cycle curve. That is, if the current state is inside the curve but different from the fixed point, then the movement is outward toward the limit cycle. If the current state is outside the curve, then the motion is inward toward the limit cycle. If the current state were on the limit cycle, the trajectory could never leave and the result would be ordinary periodic motion. If the current state were on the equilibrium point, no motion at all occurs; that is, we are sitting on a critical point, or point of equilibrium.

Limit-cycle behavior can sometimes be "evolved" from a system possessing a single fixed point by a parameter change that leads to a "catastrophe" in the sense that the global behavior of the system is altered. In the present case, the system is transmogrified into one with the new property of possessing a limit cycle. Such an evolutionary catastrophe is called a Hopf bifurcation (see, e.g., Beltrami, 1987). A bifurcation occurs by way of the single equilibrium splitting into itself plus a limit cycle.

Figure 5 shows the same stage variables as in Figure 3, but for four distinct starting values of distance from goal. It appears that there could be a different limiting cycle for each starting point, a type of behavior very different from the classical limit-cycle dynamic. Note that as the distance start position is closer to the goal, the excursions away from the goal also become smaller. Furthermore, the satiation part of the consumption/satiation variable (i.e., the departure to the left of the trajectories) is less extreme, which might be anomalous in a biological mechanism. Finally, it appears that for each successively smaller initial-distance condition, the motivation variable, on the axis perpendicular to the page, may not be growing as large. In each case, moreover, the motivational aspect is clearly changing from cycle to cycle, again not appearing to reach as large a maximum each time.

This type of behavior, with distinct initial conditions leading to apparently different limit cycles (although computer approximations such as those in PHASER can never "prove" their existence), also occurred with other variables in our motivational system. For instance, choosing different starting values for consumption produced this mode of activity.

Figure 6 exhibits the ability to plot several integral curves (remember, these are plots of state variables with time explicit) with differing initial conditions or parameters on the same graph. This time, the purpose is to contrast early- and late-term behavior. Here we see that for the distance-from-goal variable, $d$, although there is considerable periodicity, it is not complete. In fact, as time grows larger, the excursions away from the goal become smaller. Furthermore, one can see from the relation of the two functions that less and less time is spent away from the goal. That is, our subject gets "hungry" more often. Hence, it may be that we do not have true limit-cycle behavior from our model. More computer experimentation and preferably some analytic work can help to decide the case one way or the other. The good news is that PHASER revealed this potential snag in the limit-cycle hypothesis, too.

The final demonstration focuses on the rotational and perspective abilities of PHASER. These are more dramatic 


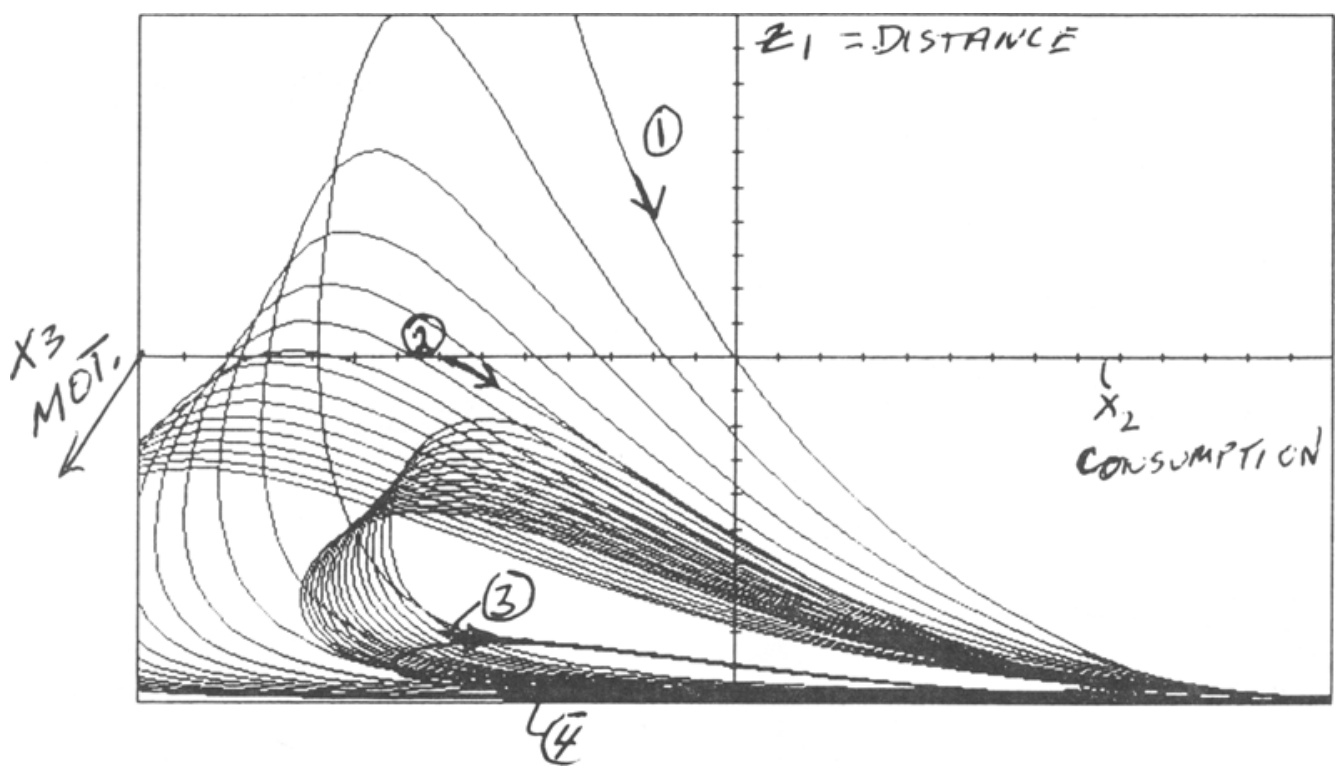

Figure 5. Again we see motivation, consumption/satiation, and distance from gaal plotted in phase space, but now four distinct initial conditions are employed and plotted in the same picture.

than our own system when we use a pair of coupled linear oscillators operating in four dimensions. Even though the system is linear, the overall behavior is surprisingly complex. The equations of the system are $d x_{1} / d t=a x_{3}$, $d x_{2} / d t=b x_{4}, d x_{3} / d t=-a x_{1}$, and $d x_{4} / d t=-b x_{2}$. Observe the extreme simplicity of these functions. This system can readily be solved analytically, but the computer still helps to reveal the complicated activity. Although there are exciting developments on the horizon regarding four-dimensional graphics (e.g., Hanson \& Heng, 1991), PHASER, like most over-the-counter graphics systems, projects the four dimensions down to three. The resulting forms are known as Lissajous figures, the side views of the total activity (see Harmoscil in PHASER). Figure 7a shows a three-dimensional trajectory in its standard form within the program, and Figure $7 \mathrm{~b}$ shows the same plot with a $90^{\circ}$ rotation about the $x$-axis. Notice that from the original orientation, the view suggests a cylinder whose "walls" are thin. This is far from true, as we see in Figure $7 \mathrm{~b}$, where we easily perceive an elliptical doughnut. All the crisscrosses are, of course, the actual trajectory weaving in and out. It may appear that it intersects itself, but this is impossible, because then it would have to return to that point of intersection again and again and

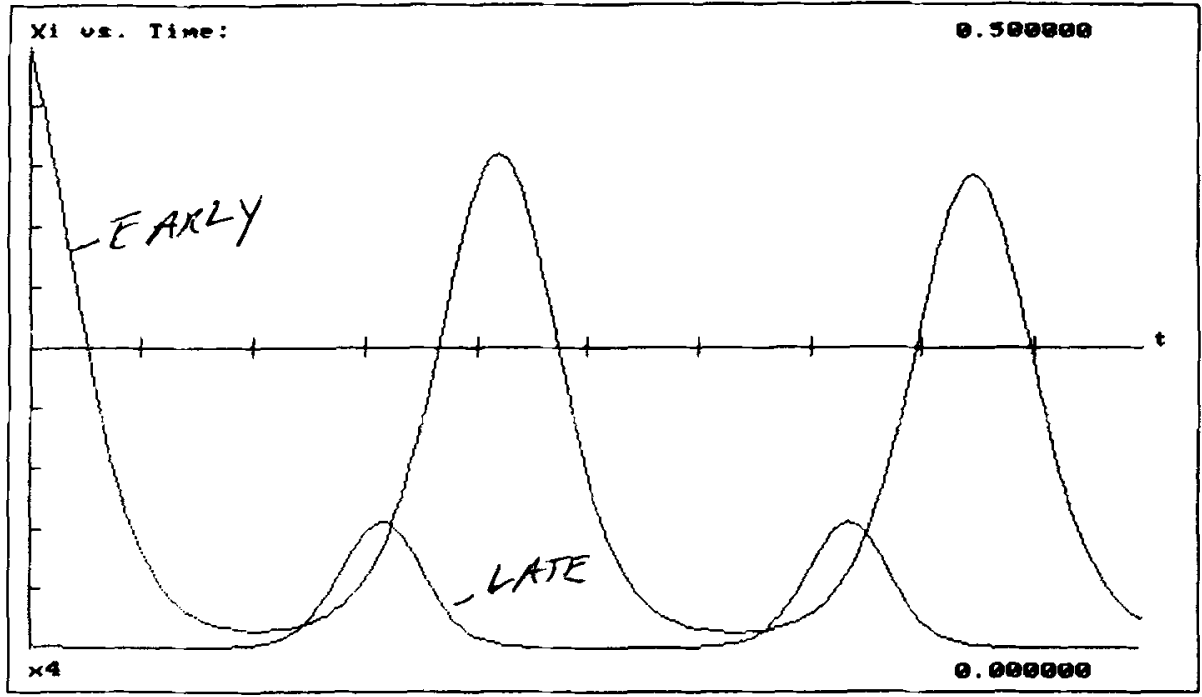

Figure 6. Two inlegral curves for distance from goal plotted for an early block of time and a much later, equal-interval block of time. Note the regularity but also the change in the magnitudes of excursion. 
(a)

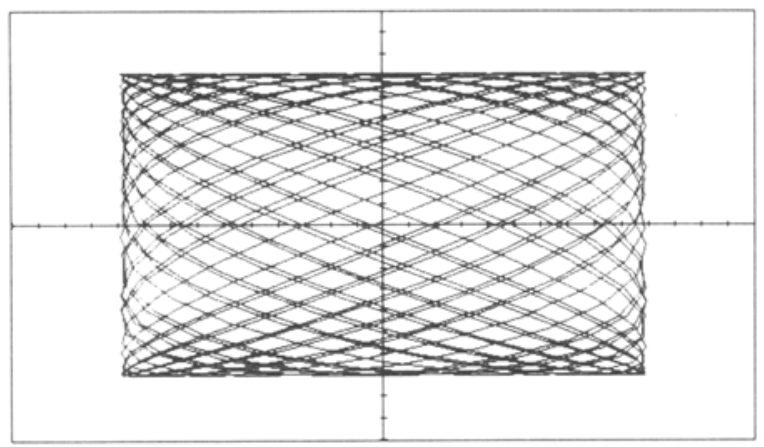

(C)

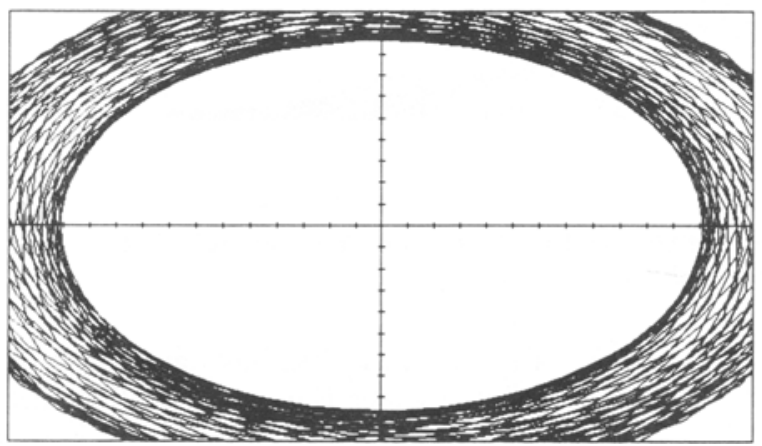

(b)

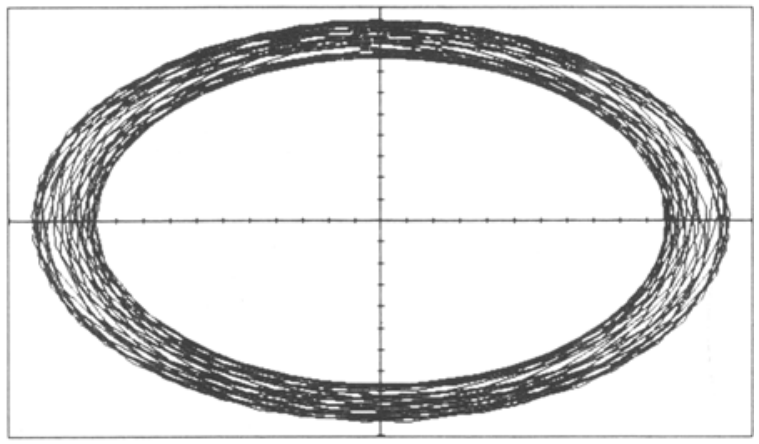

(d)

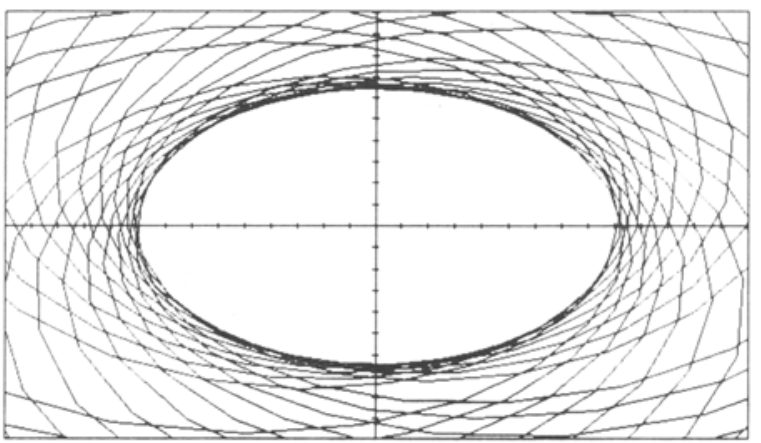

Figure 7. The complicated behavior of coupled linear oscillators, and rotation and perspective shift with PHASER (taken from Harmoscil in PHASER). (a) The resulting Lissajous figure shown without rotation or perspective shift. The remaining figures show the Lissajous figure with a $90^{\circ}$ rotation around the $x$-axis and (b) a perspective shift $=5.0$, (c) a perspective shift $=3.0$, and (d) a perspective shift $=1.0$.

would, in fact, be periodic. Figures $7 \mathrm{c}$, and $7 \mathrm{~d}$ exhibit PHASER's facility of changing perspective. In this case, we are "marching through" the center of the doughnut.

This concludes the illustration of the investigation of dynamic systems with PHASER. I have not been exhaustive; there are a number of other things PHASER can do. For instance, Poincaré plots take a cross-section plane at an arbitrary orientation and depict the pinpoint each successive "cycle" of the trajectory makes when it intersects the plane. The pattern of the pinpoints can be exceedingly instructive with quite complex dynamical systems. Another useful facility is the ability to actually watch the flow of several trajectories from distinct initial conditions simultaneously.

As noted, the present discussion corresponds to an early and actual phase of system investigation. Although the coarse behavior of MOT appears in line with our intuitions, intriguing puzzles have already cropped up. Much more computer experimentation must be done and supplemented with analytic work to attain a deeper understanding of the model's behavior. Whether even the qualitative form of the equations will stand the test of intensive probing remains to be seen. Certainly tools like
PHASER are worth their weight in gold in making that goal not only easier to reach, but often changing an impossible task to a feasible one.

\section{REFERENCES}

Beltrami, E. (1987). Mathematics for dynamic madeling. New York: Academic Press.

Busemeyer, J., Townsend, J. R. (1990). Decision field theory: A dynamic-cognitive approach to decision making. Manuscript submitted for publication.

Busemeyer, J., Townsend, J. T. (in press). The fundamental derivations from decision field theory. Journal of Mathematical Social Sciences.

DeVANeY, R. (1986). An introduction to chaotic dynamical systems. New York: Addison-Wesley.

Hanson, A. J., Heng, P. A. (1991). Visualizing the fourth dimension using geometry and light. In Proceedings of IT Visualization 91 (pp. 321-328). San Diego, CA.

Hirsch, M. W., Smale, S. (1974). Differential equations, dynamical systems, and linear algebra. New York: Academic Press.

HuLL, C. L. (1938). The goal gradient hypothesis applied to some "field force" problems in the behavior of young children. Psychological Review, 45, 271-299.

KoçAK, H. (1989). Differential and difference equations through computer experiments (2nd ed.). New York: Springer-Verlag 
LEWIN, K. (1935). A dynamic theory of personality. New York: McGraw-Hill

LUENBERGER, D. G. (1979). Introduction to dynamic systems. New York: Wiley.

Miller, N. E. (1959). Liberalization of basic S-R concepts: Extensions to conflict behavior, motivation and social learning. In S. Koch (Ed.), Psychology: A study of a science (Vol. 2, pp. 196-292). New York: McGraw-Hill.

SIBIRSKY, K. S. (1975). Introduction to topological dynamics. Leiden, The Netherlands: Noordhoff.

TOWNSEND, J. T. (1988). A neuroconnectionistic formulation of dynamic decision field theory. In D. Vickers (Chair), Symposium on decision and control mechanisms in perception and memory. 24th Intemational Congress of Psychology, Sydney, Australia.

Townsend, J. T. (1991). Phasing into PHASER. Behavior Research Methods, Instruments, \& Computers, 23, 77-78.

TOWNSEND, J. T., ASHBY, F. G. (1983). The stochastic modeling of elementary psychological processes. Cambridge: Cambridge University Press.

TownSEnd, J. T., \& Busemeyer, J. R. (1989). Approach-avoidance: Return to dynamic decision behavior. In C. Izawa (Ed.), Current Issues in Cognitive Processes: Tulane Floweree Symposium on Cognition (pp. 107-133). Hillsdale, NJ: Erlbaum. 